\title{
SOS save our surgeons: Stress levels reduced by robotic surgery
}

\author{
A. M. Hurley ${ }^{1}$ • P. J. Kennedy ${ }^{2}$ - L. O'Connor ${ }^{1}$ - T. G. Dinan ${ }^{2}$ • \\ J. F. Cryan ${ }^{2}$ - G. Boylan ${ }^{3}$ - B. A. O'Reilly ${ }^{1}$
}

Received: 18 September 2014 / Accepted: 13 April 2015 /Published online: 16 May 2015

(C) Springer-Verlag Berlin Heidelberg 2015

\begin{abstract}
Robotic-assisted laparoscopic surgery (RALS) is making an increasingly significant contribution to the field of gynaecological surgery. RALS offers similar patient benefits to standard laparoscopic surgery (SLS) with a potentially more ergonomically friendly and less stressful environment for the surgeon. However, our understanding of how RALS may potentially reduce physiological stress on the surgeon is currently limited. To assess how performing surgical tasks using RALS in comparison to SLS impacts on hypothalamic pituitary adrenal (HPA) axis function and sympathetic nervous system (SNS) activity, two key indicators of the physiological stress response. This study is an analytical, within subjects, crossover design study. Sixteen surgically inexperienced medical students performed tasks with both SLS and RALS instrumentation. Blood pressure (BP) was taken before and after task performance. Skin conductance level (SCL), heart rate (HR) and HR variability (HRV) were measured continuously during task performance. Pre- and post-task saliva samples were collected to determine cortisol levels using ELISA. SCL was significantly lower during RALS in comparison to SLS task performance $(p<0.05)$. HR was significantly lower during RALS vs. SLS tasks $(p<0.01)$. Both HRV measures were significantly higher during RALS vs. SLS tasks $(p<0.01)$. Cortisol levels and BP were lower during RALS vs. SLS but did not reach
\end{abstract}

A. M. Hurley

aoife.hurley@umail.ucc.ie

1 Department of Urogynaecology, Cork University Maternity Hospital, 25 Lindville Blackrock rd, Wilton, Cork, Ireland

2 Alimentary Pharmabiotic Centre, University College Cork, Cork, Ireland

3 Department of Paediatrics \& Child Health, University College Cork, Cork, Ireland statistical significance ( $p=0.73$ and $p=0.22$, respectively). Stress can impair surgeon's technical and nontechnical skills. These results indicate that the improved ergonomic setup of RALS has a beneficial impact on physiological indicators of stress. This also demonstrates the potential of RALS to reduce the negative effects of long-term stress exposure on the surgeon.

Keywords Robotic $\cdot$ Laparoscopic $\cdot$ Minimally invasive surgery $\cdot$ Stress

\section{Introduction}

Minimally invasive techniques, both standard laparoscopic surgery (SLS) and robotic-assisted laparoscopy surgery (RALS) are rapidly becoming the standard surgical technique for many surgical procedures [1]. The patient advantages of minimally invasive surgery are clear and have been well established in the literature. These benefits include decreased postoperative pain with reduced analgesic requirements, faster recovery times, reduced wound complications, improved cosmesis and lower morbidity $[2,3]$. However, the patient benefits of SLS are at an ergonomic cost to the surgeons performing these procedures as they are exposed to a number of potential occupational hazards [4]. The ergonomics in SLS are poor due to limited two-dimensional vision of the surgical field which impairs depth perception, an unstable camera held by an assistant of varying levels of competency, limited degrees of freedom of straight laparoscopic instruments and movements being counterintuitive (i.e. moving the instrument to the right produces a movement to the left on the viewer). Such factors are known to exert much greater stress on the surgeon, both cognitively and physiologically, during SLS when compared to open surgical procedures $[5,6]$. One solution to this problem is to use robotic-assisted techniques, which offer a more ergonomically friendly environment for the surgeon. 
RALS offers many ergonomic advantages for the surgeon including a 3-dimensional view of the surgical field, surgeon control of the camera held in position by a robotic arm providing a more stable platform, seven degrees of wrist movement and intuitive movements (i.e. moving the control to the right produces a movement to the right on the viewer) $[7,8]$.

To date, two studies have demonstrated that the improved ergonomics in RALS reduce sympathetic nervous system (SNS) activity and could result in a decrease in cognitive and physiological stress on the surgeon in comparison to SLS $[9,10]$. Van der Schatte et al. [9] demonstrated significant decreases in SNS arousal during RALS task performance in comparison with SLS. Root mean square of successive differences (RMSSD) between consecutive beats, average heart rate (HR) and pre-ejection period (PEP) were measured. Questionnaires were used to assess stress subjectively. These parameters all indicated lower physical stress and cognitive workload during RALS task performance.

Klein et al. [10] assessed perceived mental workload and stress levels during SLS and RALS task performance through the use of two other questionnaires. They concluded that the novice subjects experienced similar mental workload in both the SLS and RALS interfaces but stress levels were significantly lower with the RALS system.

Berguer and Smith [11] measured surgeons' mental workload through skin conductance level (SCL). They demonstrated a decrease in average SCL with the use of the RALS instrumentation, but this did not reach statistical significance. Additionally, they demonstrated the potential of RALS techniques to eliminate the reported thumb fatigue experienced by the surgeons during SLS.

Robotic-assisted surgery appears to reduce SNS activity as highlighted by the studies reviewed above. However, SCL measures described by Berguer and Smith were for a short duration not reflective of realistic operating times. In addition, electrode placement was on the surgeons' palm so recordings were susceptible to movement artifact. Therefore, no significant difference in SCL between RALS and SLS conditions has been previously described.

The impact on the function of the other key stress response system, the hypothalamic-pituitary-adrenal (HPA) axis, is unknown. Whilst the SNS comprises the immediate response to a stressor, the HPA axis is a slower secondary response. In response to stressful stimuli corticotropin- releasing hormone (CRH) is released from the paraventricular nucleus of the hypothalamus. CRH stimulates the anterior pituitary to release adrenocorticotropin-releasing hormone $(\mathrm{ACTH})$, which in turn stimulates the adrenal cortex to release cortisol, the main glucocorticoid in humans [12]. Prolonged and chronically sustained activation of the HPA axis may have long-term negative consequences [13-16] impacting on health, mood, cognition, and may lead to the development of stress-related diseases [17-19]. The full extent to which the neuroendocrine systems are activated during each of these laparoscopic interfaces is unknown as no studies to date have assessed the HPA-axis response to the two surgical conditions. However, given the negative consequences of chronic HPA-axis activation, it is of paramount importance to investigate the impact on neuroendocrine function during each surgical technique as well as further assessing the extent of SNS activation particularly in relation to SCL.

\section{Methods}

This study aimed to assess the impact on physiological stress levels whilst performing surgical tasks using SLS in comparison to using RALS instrumentation.

The objectives were to identify any differences in SNS activity measured by SCL, HR, HR variability (HRV) and BP and to assess the impact on HPA-axis function, as measured by salivary cortisol levels.

This study is an analytical, within subjects, crossover design study.

Ethical approval was granted by the Clinical Research Ethics Committee (CREC), and the study was carried out in Cork University Maternity Hospital. The sample consisted of 16 surgically inexperienced fourth year medical students from University College Cork who were recruited via email correspondence. This sample size was selected as a previous published study [9] in the area used this number, and it was manageable within the constraints of available theatre times. Interested students were required to complete a questionnaire prior to selection (Appendix B) to assess and control for factors know to affect the HPA axis including, age, gender, BMI, drugs (both elicit and prescribed) and physical activity levels [20-22]. The participants were included in the study if they were aged between 18 and 40 years, male, healthy with no history of any psychiatric conditions, not taking any medication, a non-smoker and had a BMI within normal range (18$25 \mathrm{~kg} / \mathrm{m}^{2}$ ) (see Fig. 1). We chose to include only male volunteers in the study as the female cortisol response to stress changes with the menstrual cycle [23]. All participants completed informed consent forms prior to commencement of the study (Appendix A).

Each subject carried out tasks with both the SLS and RALS instrumentation. Subjects were required to attend two separate sessions approximately 2 weeks apart. All subjects were assigned a subject number, and the order of the study visits was randomised, with half of the participants performing SLS first and the other half performing RALS first. To control for the diurnal fluctuation in cortisol [24], all study visits took place between 2 and $7 \mathrm{pm}$ and the subject's second visit was scheduled for approximately the same time as their first. Preparation guidelines for the study visits were given to all subjects (Appendix C). Subjects were required to wear surgical scrubs in both conditions in order to emulate the operating theatre environment.

The tasks were performed by each participant using the 3-Dmed ${ }^{\circledR}$ task kit, a validated training tool for laparoscopic surgery [25] (see Fig. 2). Task description (Appendix D). The participants were instructed that task 
Fig. 1 Study population demographics

\begin{tabular}{|c|c|c|c|c|}
\hline $\begin{array}{c}\text { Smoking } \\
\text { Status }\end{array}$ & Age & Sex & BMI & $\begin{array}{c}\text { Alcohol } \\
\text { Consumption } \\
\text { per week } \\
\text { (units) }\end{array}$ \\
\hline Non-smokers & $\begin{array}{c}\text { Average: } \\
24.375 \text { years } \\
\text { Range: } 21-30 \\
\text { years }\end{array}$ & Male & $23.365 \mathrm{~kg} / \mathrm{m}^{2}$ & $\begin{array}{c}\text { Average: } 5.95 \\
\text { units/week }\end{array}$ \\
\hline
\end{tabular}

Skin conductance was recorded using a Nexus-4 ambulatory monitoring device (Mind Media BVC) and disposable GSR electrodes (VERMED ${ }^{\circledR}$ ). Placement of electrodes was as recommended on the foot (Public Recommendations for Electrodermal Measurement) [28] (Appendix E [29]). Baseline SCL values were recorded for 5 min with the subject in a stationary standing position for the SLS session and in a seated position for the RALS session. SCL was recorded continuously throughout task performance. Room temperature was recorded every $30 \mathrm{~min}$ and maintained at $21-22.5{ }^{\circ} \mathrm{C}$. Outcome measurement assessed was average SCL.

HRV was measured with Meditrace $100-\mathrm{Ag} / \mathrm{ACl}$ electrodes (Kendall ${ }^{\circledR}$ ) attached to the NeXus-4 ambulatory monitoring device. HRV values were recorded for $5 \mathrm{~min}$ with the subject in a stationary standing position for the SLS session and in a seated position for the RALS session. These values were recorded continuously throughout task performance. Outcome measurements assessed were average HR, RMSSD and Standard deviation of N-N deviations (SDNN). RMSSD and SDNN are measures of HRV which is tightly linked to respiratory sinus
Fig. 2 The 3-Dmed ${ }^{\circledR}$ task kit. a Post and sleeve; $\mathbf{b}$ loops and wire; c wire chaser; $\mathbf{d}$ peas on a peg
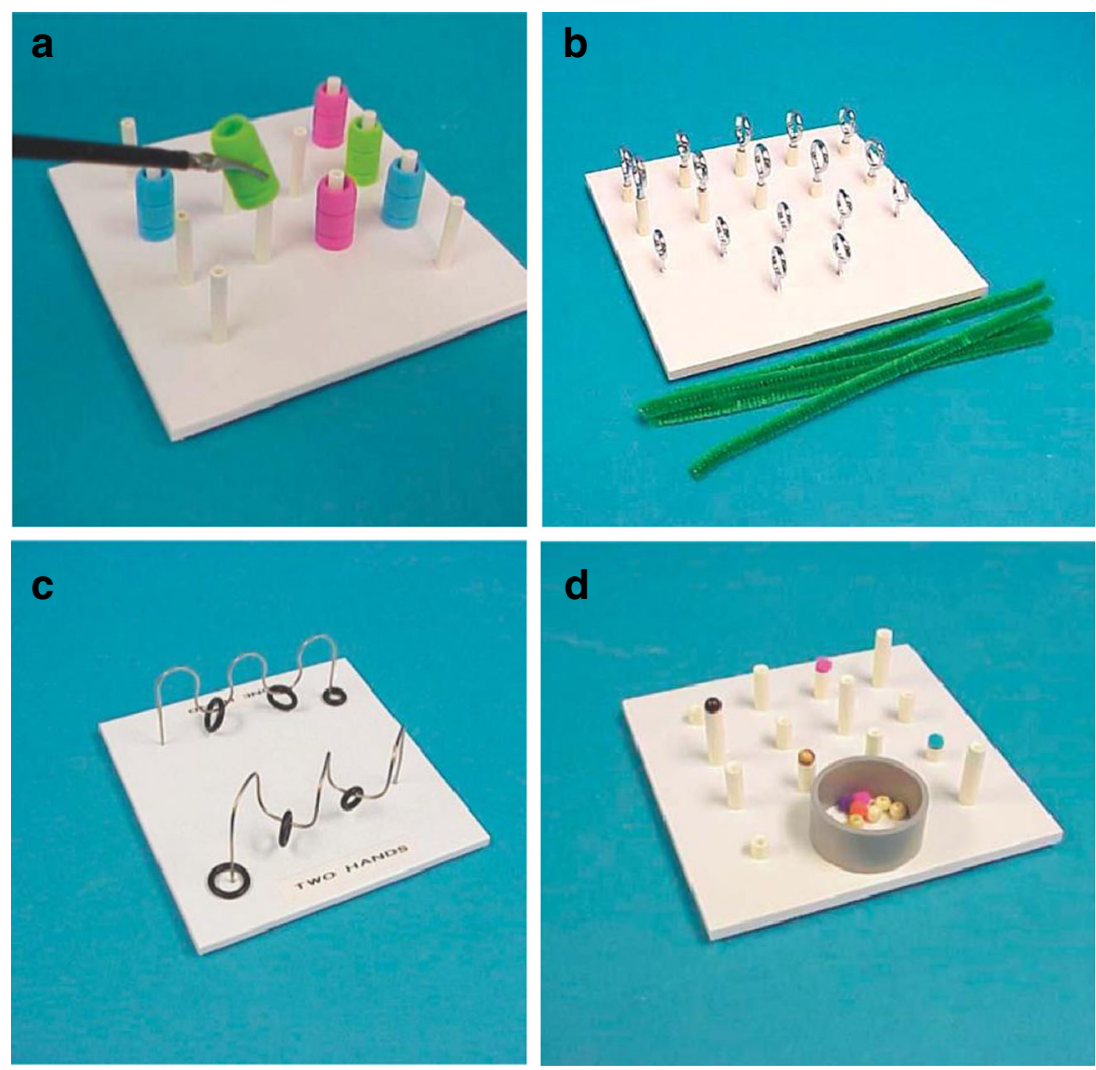
Fig. 3 A figure showing the timing of cortisol sampling, SCL and HR measurements in relation to task performance. ( $S C L$ skin conductance level, $H R$ heart rate.)
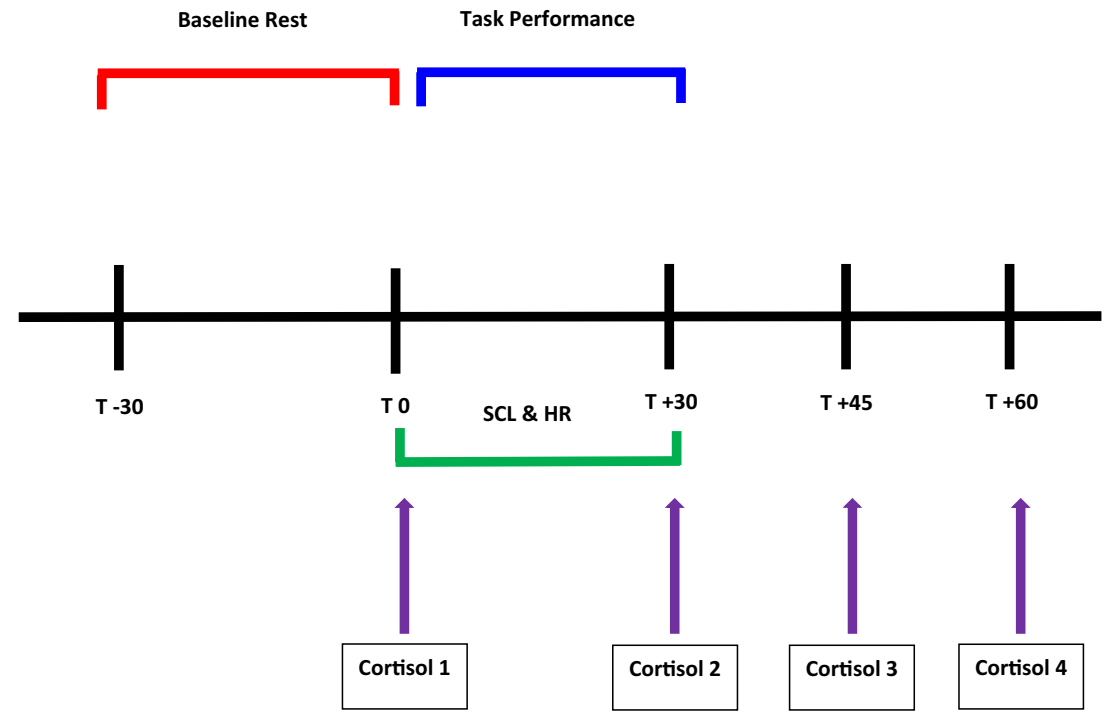

arrhythmia (RSA). Changes in RSA reflect changes in vagal activity [30]. When vagal activity decreases HRV also decreases. An increase in stress therefore leads to a decrease in HRV.

BP was measured before starting the tasks and after task completion. Outcome measurement assessed was the average of the mean arterial pressure (MAP).

Subjects rested for $30 \mathrm{~min}$ before a baseline salivary cortisol sample was taken, another sample was taken immediately after task completion and two further samples were taken at $15 \mathrm{~min}$ intervals (see Fig. 3). Samples were taken using a Salivette® (Sarstedt) device. Subjects were instructed to chew on the cotton bud for $1.5 \mathrm{~min}$ before returning it to the tube. Samples were kept at $3{ }^{\circ} \mathrm{C}$ then centrifuged at $1000 \mathrm{~g}$ for $10 \mathrm{~min}$ to extract the saliva and stored at $-80{ }^{\circ} \mathrm{C}$ before analysis. Samples were analysed using ELISA (ENZO ${ }^{\circledR}$ life sciences) as per the manufacturers' instructions. Inter and intra assay variability was less than $10 \%$.

Statistical analysis was carried out as previously described [9]. A series of paired sample $t$ tests were carried out to investigate differences between the SLS and RALS conditions for SCL, HR, SDNN, RMSSD, BP and cortisol levels, whilst performing each of the four surgical tasks. All data reported as

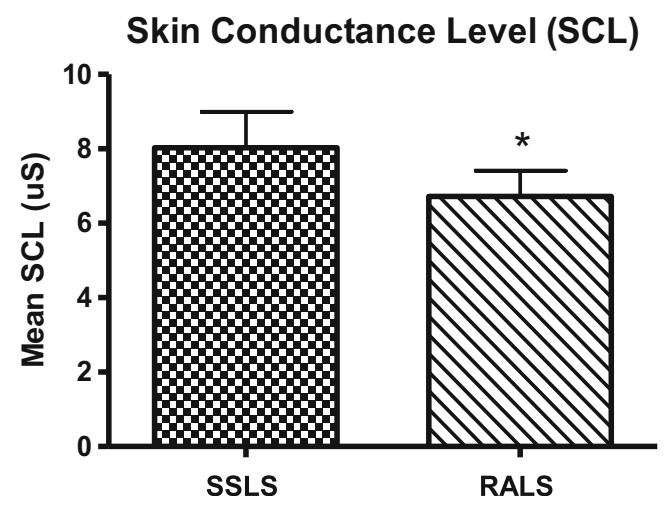

Fig. 4 Skin conductance level during SLS task performance was significantly higher than during RALS tasks $\left({ }^{*} p<0.05\right)$ mean \pm S.E.M. Differences were considered significant at an alpha level of 0.05. All statistical procedures were carried out using IBM SPSS statistics 20.0 for Windows Software package.

\section{Results}

There was a significant difference in SCL between the SLS (M= $8.03 \pm 0.96)$ and RALS $(\mathrm{M}=6.71 \pm 0.69)$ conditions $(\mathrm{t}(15)=2.45$, $p=0.027$; see Fig. 4), indicating that SNS arousal was higher during SLS task performances than during RALS task performance.

There was a significant difference in average HR between the SLS condition $(\mathrm{M}=84.41 \pm 2.7)$ and the RALS condition $(\mathrm{M}=76.37 \pm 2.37)(\mathrm{t}(15)=3.45, p=0.004$; see Fig. 5) indicating that HR was higher during standard laparoscopic task performances than during robotic-assisted task performance.

There was a significant difference in SDNN, a measure of HRV, between the SLS $(M=54.97 \pm 5.42)$ and the RALS $(\mathrm{M}=77.82 \pm 6.55)(\mathrm{t}(15)=5.38, p<0.01$; see Fig. 6) indicating greater HRV during RALS task performance than SLS.

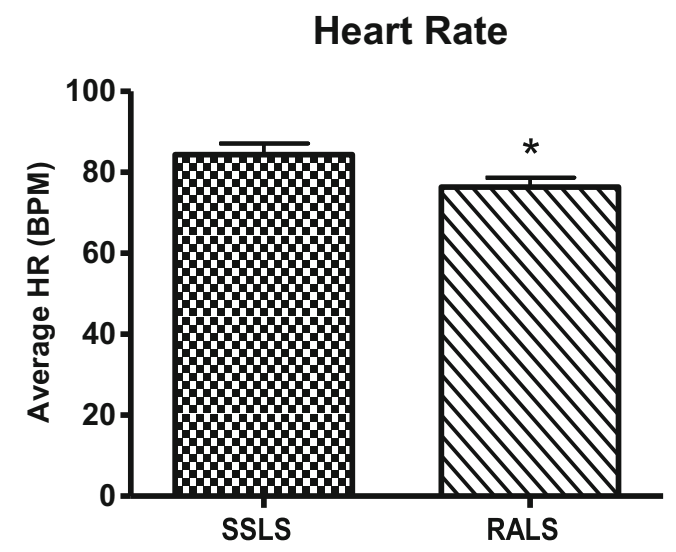

Fig. 5 There was a significant decrease in heart rate from SLS task performance to $\operatorname{RALS}(* p<0.01)$ 


\section{Standard deviation of N-N intervals} (Heart rate variability)

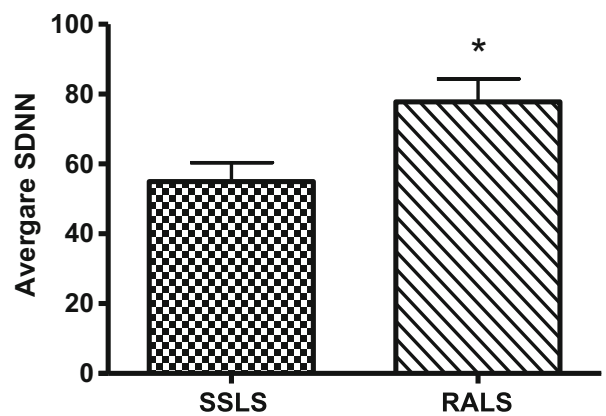

Fig. 6 SDNN was significantly higher during RALS task performance $\left({ }^{*} p<0.01\right)$

There was a significant difference in RMSSD a second measure of HRV between the SLS $(\mathrm{M}=33.0 \pm 4.90)$ and RALS ( $\mathrm{M}=$ $69.91 \pm 6.88)$ condition $(\mathrm{t}(15)=6.67, p<0.01$; see Fig. 7), indicating again that HRV was increased whilst using the RALS instrumentation when compared to the SLS instrumentation.

MAP was calculated from BP readings taken before and after task performance using the formula: $(\mathrm{MAP}=$ diastolic pressure $+1 / 3$ (systolic pressure-diastolic pressure)). Average increase in MAP was higher during SLS $(\mathrm{M}=4.23 \pm 1.34)$ than RALS ( $M=0.50 \pm 2.99)$. $(\mathrm{t}(15)=1.28, p=0.22$; see Fig. 8$)$. However, this did not reach statistical significance

Cortisol changes from baseline between SLS and RALS conditions showed a higher percentage increase in cortisol levels in the standard laparoscopy $(\mathrm{M}=17.10 \pm 16.44)$ than in the robot assisted $(\mathrm{M}=8.19 \pm 15.62)$ condition. $(\mathrm{t}(15)=0.35, p=0.73$; see Fig. 9). However, this did not reach statistical significance.

\section{Discussion}

This study aimed to assess the impact on physiological stress levels, measured by both the SNS and the HPA-axis, whilst performing surgical tasks using SLS in comparison to using RALS instrumentation.

\section{Standard deviation of N-N intervals (Heart rate variability)}

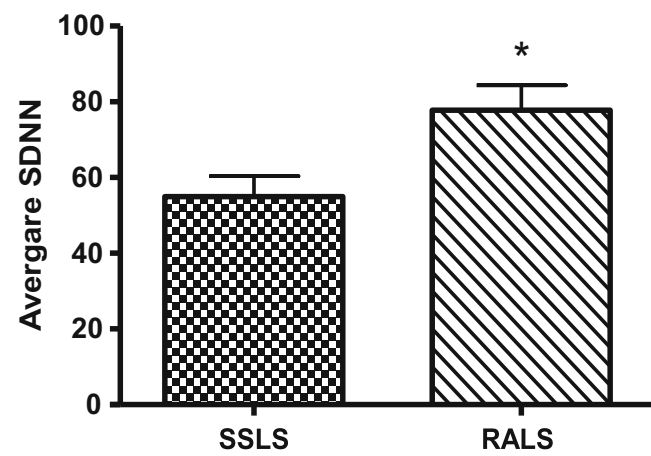

Fig. 7 RMSSD was significantly higher during RALS task performance $\left({ }^{*} p<0.01\right)$

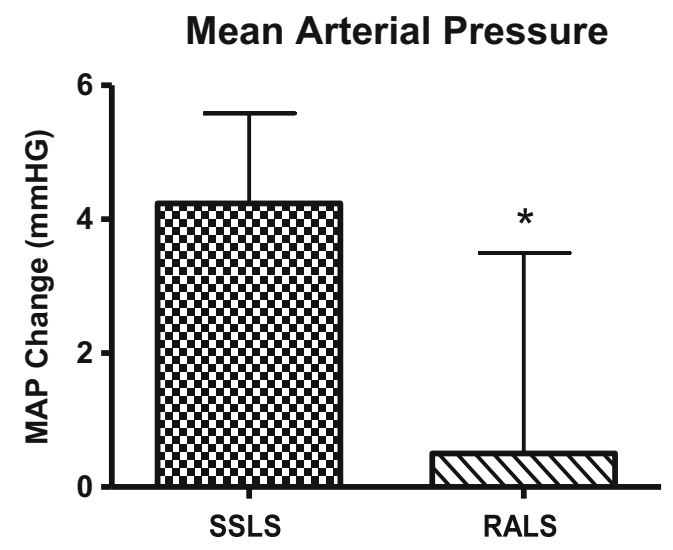

Fig. 8 The change in mean arterial blood pressure was higher during SLS than RALS task performance

SCL is a sensitive measure of SNS arousal and stress. There is a positive relationship between electrodermal lability (as measured by SCL) and beta-adrenergic myocardial reactivity to stress, particularly under conditions of task novelty or uncertainty [31]. SCL was significantly higher during task performance with the SLS than the RALS instrumentation. This new finding demonstrates lower SNS input when using the robot-assisted interface thus reflecting lower stress levels than during standard laparoscopic simulation.

We also confirmed that RMSSD described by van der Schatte et al. and SDNN (a finding not previously described) are higher during RALS than SLS. When vagal activity decreases HRV also decreases. An increase in stress will therefore lead to a decrease in HRV. Therefore, increases in HRV whilst performing RALS tasks reflect lower levels of SNS input and therefore lower stress levels.

This study confirmed previous reports [9] that average HR is significantly higher whilst performing tasks with SLS in comparison to RALS. Increased HR once again reflects higher levels of SNS arousal. Therefore, the lower average HR recorded during RALS task performance indicates lower stress levels whilst using the robotic interface.

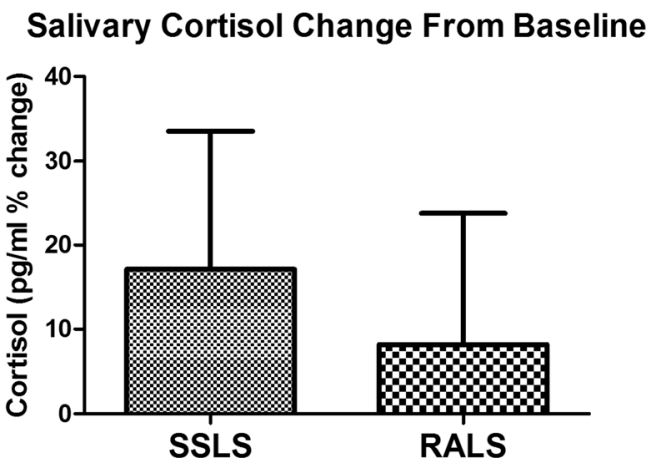

Fig. 9 The increase in cortisol levels was higher during SLS vs. RALS task performance 
Another measure of SNS arousal is blood pressure. This measurement has not previously been described in relation to SLS and RALS. Average MAP values during RALS task performance were lower than during SLS task performance. However, these values did not reach statistical significance. This lack of statistical significance may once again reflect the relatively short "operating time".

This is the first time salivary cortisol measures have been applied in ergonomic surgical research, and it is a strategy which has provided some novel and interesting findings. Cortisol is an easily accessible peripheral measure that provides a reliable indication of HPA axis dysfunction [32]. Cortisol levels were higher during SLS task performance than RALS; however, these results did not reach statistical significance. An important point to consider here is that the "operating time" i.e. the duration of task performance was shorter than a real surgical procedure, so speculatively cortisol levels may increase more under real surgical conditions. Regardless of this if cortisol levels are always elevated to a certain degree when performing laparoscopic surgery, this may impact negatively on the surgeon as prolonged exposure to excess cortisol could result in damage to the hippocampus and permanent memory impairment as well as stress-related diseases [13-19].

These results are all highly suggestive of stress reduction with robotic assistance. However, some critical remarks must be made. Firstly, this study was carried out under simulated, experimental conditions. Stressful or distracting factors that may be present in a real life theatre setting were absent in this experimental setup; therefore, these results may not be directly comparable with stress in actual surgical situations. Secondly, throughout the RALS task performance, subjects remained in a seated position and this may have influenced our results; however, this does reflect the surgeons position during actual robotic surgery. The fixed table height for all participants during the SLS tasks compared to the adjustable stool height and robotic arm position during the RALS tasks may also have impacted the results.

Thirdly, the task performance time (approximately $30 \mathrm{~min}$ ) was significantly shorter than standard operating time. It is likely that the surgeons would experience greater levels of stress and fatigue whilst performing longer procedures.

The subjects included in this study were all male, and this single gender factor is a major limitation of the study. We chose to exclude females as the female stress response changes throughout the menstrual cycle [23]. This was in order to avoid complications of controlling for menstrual cycle in a repeated measures study where a 1 month gap would have to be left to make sure females were always tested at the same menstrual phase.

Finally, all subjects included in this study were surgically inexperienced and it is unclear whether levels of experience might influence the stress response. Therefore, it is difficult to determine if the study population is comparable with a population of laparoscopic surgeons. Future studies could assess if these physiological stress parameters are similarly affected in experienced surgeons under real surgical conditions.

The aforementioned factors may have influenced our results to a certain extent; however, our results clearly indicate that the improved ergonomic setup of RALS leads to lower physiological indicators of stress in this study population. These results provide early indicators that the employment of RALS equipment could decrease surgeon fatigue and reduce the stress that has been shown to affect both the technical [33] and nontechnical (communication, teamwork and decision making) skills [34-37] of the surgeon. A reduction in the stress that impairs these fundamental skills could therefore improve surgical outcomes for patients as well as decreasing the negative effects of long-term stress exposure on the surgeon.

Declaration of competing interests Dr. O'Reilly is a proctor for Intuitive Surgical; there are no other relationships or activities that could appear to have influenced the submitted work. This research received no specific grant from any funding agency in the public, commercial or not-for-profit sectors.

The lead author affirms that the manuscript is an honest, accurate and transparent account of the study being reported; that no important aspects of the study have been omitted; and that any discrepancies from the study as planned have been explained.

Ethical approval was granted by the Clinical Research Ethics Committee (CREC), and the study was carried out in Cork University Maternity Hospital. All participants were required to complete informed consent forms prior to commencement of the study (Appendix A).

Contributors Dr. Aoife Hurley contributes in study design and planning, subject recruitment, data collection, laboratory sample analysis, statistical analysis, reporting of the study.

Paul J Kennedy contributed in study design and planning, subject recruitment, data collection, laboratory sample analysis, statistical analysis, reporting of the study.

Lorraine O'Connor contributed in data collection.

Dr. Barry A. O'Reilly contributed in study design and planning, reporting of the study.

Professor Geraldine Boylan contributed in study design and planning.

Professor Timothy G. Dinan contributed in study design, provision of laboratory facilities and equipment.

Professor John F. Cryan contributed in study design, provision of laboratory facilities and equipment.

Guarantor Dr. Barry A. O’Reilly

\section{Appendix A}

\section{SUBJECT INFORMATION SHEET}

Study Title: A study to assess stress levels in straight stick versus robotic assisted laparoscopic surgery.

Protocol No.:

Principal Investigators: Dr. Barry O’Really

Aoife Hurley 


\section{Co- Investigators:}

Site of Investigation: Cork University Maternity Hospital (CUMH),

Why is this study being run?

You are invited to participate in a research study to assess changes in stress levels whilst peforming tasks with both a laparoscopic box trainer and DaVinci Robot.

\section{Study procedures}

If you agree to participate in the study you will be asked to visit the study site clinic at Cork University Maternity Hospital. Participation will involve 2 visits. This study will involve up to 20 subjects. All other subjects will be males between 18 and 40 years of age.

\section{Visit 1 Outline}

You will be advised of the purpose of the study and the procedures which will be undertaken. You will be given a copy of the Subject Information sheet, which will explain what is required from you. If interested, you will then be requested to read and sign the Informed Consent form, and receive a signed copy.

You will be asked some general questions concerning your health and personal details. Details of your current and/or past medications will also be collected.

Two electrodes to measure perspiration will be attached to your ankle and you will be asked to rest for $30 \mathrm{~min}$. After this time you will be asked to perform a series of tasks with one of the surgical trainers, you will only be informed of what is required at the beginning of each stage this is to insure that you are not able to prepare for the procedure which may affect the results. Before, during and after the tasks you will provide a total of 4 saliva samples which will be assessed for a stress hormone called cortisol.

\section{Visit 2 Outline}

You will return for visit 2 one week later-the second visit will have the same format as visit 1. Upon arrival you will have two electrodes attached to your ankle and be asked to rest for $30 \mathrm{~min}$. After this time you will be asked to perform a series of tasks with the other surgical trainer, you will only be informed of what is required at the beginning of each stage this is to insure that you are not able to prepare for the procedure which may affect the results. Before, during and after the tasks you will provide a total of 4 saliva samples which be assessed for a stress hormone called cortisol.

What happens if I start the study and change my mind later?

You do not have to take part in the study, participation is entirely voluntary. Refusal to participate, or discounting participation at any time, will involve no penalty, loss of benefits or denial of treatment or services by the Cork Teaching Hospital.

\section{Will I experience any unpleasant side effects?}

\section{Confidentiality.}

All the information gathered from this study will be stored on a computer or paper files and will be treated confidentially. You will be identified only by a subject number. In the event

of any publication regarding this study, your identity will not be disclosed.

What happens if there is anything I do not understand?

If there is anything you are not sure about, I will be happy to explain anything in more detail to you. The study will be fully explained to you before you decide if you want to take part.

CONSENT BY SUBJECT FOR PARTICIPATION IN RESEARCH PROTOCOL

Protocol Number:

Patient Name:

Title of protocol: A study to assess stress levels in straight stick versus robotic assisted laparoscopic surgery.

\begin{tabular}{|}
$\begin{array}{r}\text { Principal Investigator(s): Dr. Barry O’Reilly, } \\
\text { Aoife Hurley: aoife.hurley@umail.ucc.ie } \\
\text { +353863455013 }\end{array}$ \\
\hline
\end{tabular}

Participation in this study is voluntary and you may withdraw at any time for any reason

The research project and procedure associated with it have been fully explained to me. All experimental procedures have been identified and no guarantess have been given about the possible results. I have had the opportunity to ask questions concerning any and all aspects of the project and any procedures involved. I am aware that participation is voluntary and I may withdraw consent at any time. I am aware that my decision not to participate or to withdraw will not restrict my access to health care services normally available to me. Confidentially of records concerning my involvement in this project will insurance as is required by law in the event of injury resulting from this research.

I, the undersigned, hereby consent to participate as a subject in the above described project conducted at the Cork University Maternity Hospital. I have received a copy of this consent form for my records. I understand that if I have any questions concerning this research, I can contact the investigator listed above. If I have further queries concerning my rights in connection with the research, I can contact the Clinical Research Ethics Committee of the Cork Teaching Hospitals, Lancaster Hall, 6 Little Hanover Street, Cork.

After reading the entire consent form, if you have no further questions about giving consent, please sign where indicated.

Subject's Signature:

NAME (BLOCK LETTERS):

Investigator's Signature:

NAME (BLOCK LETTERS)

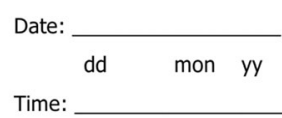

Date:

dd mon yy 


\section{Appendix B}

A study to assess stress levels in straight stick versus robotic assisted laparoscopic surgery.

\section{Screening Questionnaire}

Please fill in the following details by marking the appropriate yes or no answer with an $\mathbf{X}$ or by filling in the required details in the space provided (please be honest as these details will be verified before entry to the study):

* Your email address:

1. Age:

2. Gender: Male Female

3. Are you a smoker? (if yes please indicate how many per day):

Yes No

5-10 per day $\quad$ 10-20 per day over 20 per day

4. What is your height and weight? Height $(\mathrm{cm})$ Weight $(\mathrm{kg})$

5. Do you drink alcohol? (if yes please indicate how many units per week; 1 unit= half pint of ordinary strength beer/ $25 \mathrm{ml}$ measure of spirits. 1.5 units= small $(125 \mathrm{ml})$ glass of ordinary strength (12\%) wine:

Yes No

$0-5$ units per week 5-10 per week 10-15 per week 15-20 per week

$20+$ per week

6. Do you suffer from any acute or chronic illness? For example; cardiovascular, gastrointestinal (e.g. Inflammatory Bowel Disease)or immunological: Yes No

7. Do you suffer from any psychiatric disorders? For example; depression, anxiety disorders, bipolar spectrum disorders, schizophrenia or any other not mentioned? Yes No

8. Are you currently taking any antidepressant, anti-psychotic, anti-anxiety, steroidal or opioid based pain relieving medications?

Yes No

9. Have you been receiving any experimental drugs as part of a recent experimental trial in the past 30 days? Yes No

Please save completed questionnaire and send as an attachment to: Aoife.hurley@umail.ucc.ie

\section{Subject No:}

\section{Subject initials:}




\section{Appendix C}

A study to assess stress levels in straight laparoscopic surgery versus robot assisted surgery.

\section{Preparation Guidelines for Study Visit}

Day Prior To Vist:

- No strenuous exercise from $\mathbf{2}$ pm (24 h before your visit)

- No alcohol from 2 pm (24 h before your visit)

\section{Day of Visit:}

- No caffeinated beverages or caffeine containing products (e.g., tea/coffee/coke/red bull), this is includes no caffeine with breakfast.

- Lunch must be finished at least $2 \mathrm{~h}$ prior to scheduled visit time. For example if your visit is at $\mathbf{2} \mathbf{~ p m}$ then lunch must be finished by 12 .

- After lunch, no other food or beverages apart from water can be consumed in this time.

- Again, no strenuous exercise on the day of your visit (as well as from $2 \mathrm{pm}$ the day prior).

If any details are not clear please call me on 0863455013.

\section{Appendix D}

\section{Instructions}

You will receive one-minute practise time to get used to the equipment.

You will then perform 4 different exercises.

You will have $30 \mathrm{~s}$ practise time before each.

Perform each exercise for $5 \mathrm{~min}$.

Perform as many repetitions of the task as possible.

Task A

Post and sleeve:

Pick up the sleeve and pass it to it's mirrored post on the other side. Once all sleeves have been transferred repeat the exercise to transfer them back to the original side.

Error $=$ dropping the sleeve

\section{Task B}

\section{Loops and Wire:}

Pass one of the pipe cleaners through the back row of hoops form right to left. Pass the other pipe cleaner through the second row of hoops. Pass the rope through the third row of hoops (ignore the front two hoops). Pull the pipe cleaners or rope through the other side when you complete all 3 rows.

Error=passing the pipe cleaner/rope outside the next loop.
Task C

Pea on a Peg:

Move the peas from the cup to a post.

Once completed move the peas back to the cup.

Error $=$ dropping a pea

Task D

Wire Chaser:

One handed exercise: Begin with the largest ring and pass it over the wire from one end to the other while keeping it perpendicular to the wire and not touching the wire.

-As it goes over the loops you will have to rotate your wrist to keep the ring perpendicular.

Continue until you have moved all rings to the opposite side.

Error=dropping the ring

\section{Appendix E}

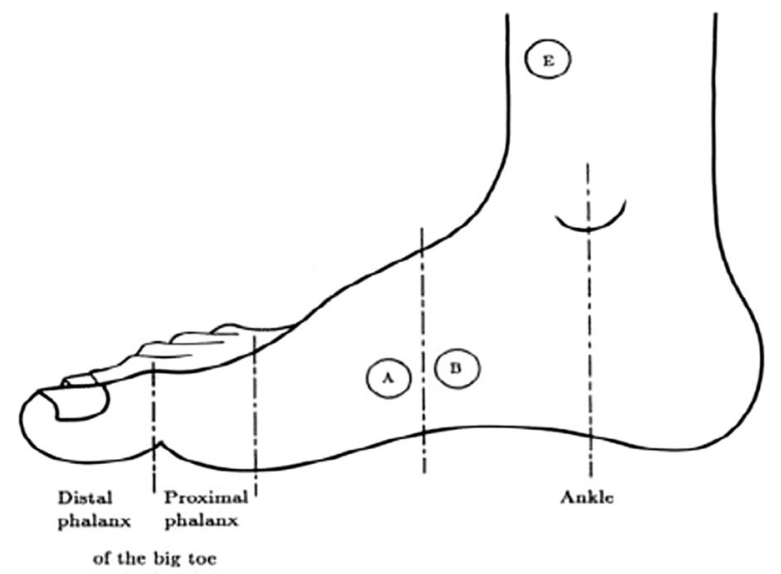

Publication recommendations for Electrodermal Measurement.

Correct positioning of electrodes (Points A \& B) for measurement of skin conductance level

\section{References}

1. Gomez G (2004) Emerging technology in surgery: Informatics, electronics, robotics. In: Townsend CM, Beauchamp RD, Evers BM et al (eds) Sabiston textbook of surgery. Elsevier Saunders, Philadelphia

2. Kenngott HG, Nickel F, Rom J, Rassweiler J, Muller-Stich BP (2012) Status of robotic assistance-a less traumetic and more accurate minimally invasive surgery? Langenbeck's Arch Surg 397: 333-341

3. Ballantyne GH (2002) The pitfalls of laparoscopic surgery, teleprescence, and telementoring. Review of early clinical results. Surg Endosc 16:1389-1402

4. Berguer R, Forkey D, Smith W (1999) Ergonomic problems associated with laparoscopic surgery. Surg Endosc 13:466-468 
5. Park A, Lee G, Seagull FJ, Meenaghan N, Dexter D (2010) Patients benefit while surgeons suffer: an impending epidemic. J Am Coll Surg 210(3):306-313

6. Berguer R, Smith WD, Chung YH (2001) Performing laparoscopic surgery is significantly more stressful for the surgeon than open surgery. Surg Endosc 15(10):1204-1207

7. Balantyne GH (2002) Robotic surgery, telerobotic surgery, teleprescence, and telementoring. Review of early clinical results. Surg Endosc 16:1389-1402

8. Dakin GF, Gagner M (2003) Comparison of laparoscopic skills performance between standard instruments and two surgical robotic systems. Surg Endosc 17:574

9. van der Schatte Olivier RH, Van't Hullenaar CD, Ruurda JP, Broeders IA (2009) Ergonomics, user comfort, and performance in standard and robot-assisted laparoscopic surgery. Surg Endosc 23(6):1365-1371

10. Klein MI, Warm JS, Riley MA (2012) Mental workload and stress perceived by novice operators in the laparoscopic and robotic minimally invasive surgical interfaces. J Endourol 26(8):1089-1094

11. Berguer R, Smith W (2006) An ergonomic comparison of robotic and laparosocpic technique: the influence of surgeon experience and task complexity. J Surg Res 134:87-92

12. Schimmer BP, Parker KL (1996) Adrenocorticotropic hormone; adrenocortical steroids and their analogues; inhibitors of the synthesis and actions of adrenocortical hormones. In: Hardman JG, Limbird LE, Molinoff PB et al (eds) The Phatmacological basis of therapeutics, 9th edn. McGraw-Hill, New York, pp 1459-1485

13. Heim C, Ehlert U, Hellhammer DH (2000) The potential role of hypocortisolism in the pathophysiology of stress-related bodily disorders. Psychoneuroendocrinology 25:1-35

14. Southwick SM, Yehuda R, Wang S (1998) Neuroendocrine alterations in posttraumatic stress disorder. Psychiatr Ann 28(8):436-445

15. Sapolsky RM (2000) Glucocorticoids and hippocampal atrophy in neuropsychiatric disorders. Arch Gen Psychiatry 57(10):925-935

16. Breier A (1989) Experimental approaches to human stress research: assessment of neurobiological mechanisms of stress in volunteers and psychiatric patients. Biol Psychiatry 26(5):438-462

17. McEwen BS (1999) Stress and hippocampal plasticity. Annu Rev Neurosci 22:105-122

18. Sapolsky RM, Armanini M, Packan D, Tombaugh G (1987) Stress and glucocorticoids in aging. Endocrinol Metab Clin N Am 16(4): 965-980

19. Lupien SJ, de Leon M, De Santi S (1998) Cortisol levels during human aging predict hippocampal atrophy and memory deficits. Nat Neurosci 1(1):69-73

20. Kirschbaum C, Kudielka BM, Gaab J, Schommer NC, Hellhammer DH (1999) Impact of gender, menstrual cycles, and oral contraceptives on the activity of the hypothalamus-pituitary-adrenal axis. Psychosom Med 61:154-162

21. Mokrani MC, Duval F, Crocq MA, Bailey P, Macher JP (1997) HPA axis dysfunction in depression: Correlation with monoamine system abnormalities. Psychoneuroendocrinology 22:63-68
22. Heim C, Newport DJ, Bonsall R, Miller AH, Nemeroff CB (2001) Altered pituitary-adrenal axis responses to provocative challenge tests in adult survivors of childhood abuse. Am J Psychiatry 158(4):575-581

23. Kirschbaum C, Pirke KM, Hellhammer DH (1993) The 'Trier Social Stress Test' - a tool for investigating psychobiological stress responses in a laboratory setting. Neuropsychobiology 28(1-2):76-81

24. Clow A, Thorn L, Evans P, Hucklebridge F (2004) The awakening cortisol response: Methodological issues and significance. Stress 7: 29-37

25. Schreuder HW, van den Berg CB, Hazebroek EJ et al (2011) Laparoscopic skills training using inexpensive box trainers: which excercises to choose when constructing a validated training course. BJOG 118(13):1576-1584

26. Narula VK, Watson WC et al (2007) A computerized analysis of robotic versus laparoscopic task performance. Surg Endosc 21(12): 2258-2261

27. Kengott HG, Muller-Stitch BP, Reiter MA, Rassweiler J, Gutt CN (2008) Robotic suturing: Technique and benefit in advanced laparoscopic surgery. Minim Invasive Ther Allied Technol 17(3):160-167

28. Edelberg R (1967) Electrical properties of the skin. In: Brown CC (ed) Methods in psychophysiology. Williams \& Wilkins, Baltimore, pp 1-53

29. Boucsein W (2012) Electrodermal activity, 2nd edn. Springer, New York, p 107

30. Berne MR, Levy MN, Stanton BA, Koeppen BM (2006) In: Berne MR, Levy MN (eds) Principles of physiology, 4th edn. Elsevier Mosby, Philadelphia, pp 383-385

31. Kelsey RM (1991) Electrodermal lability and myocardial reactivity to stress. Psychophysiology 28(6):619-632

32. Baum A, Grunberg N (1997) Measurement of stress hormones. In: Cohen S, Kessler RC, Gordon LU (eds) Measuring stress: a guide for health and social scientists. Oxford University Press, New York, pp 175-192

33. Moorthy K, Munz Y, Dosis A, Bann S, Darzi A (2003) The effect of stress inducing conditions on the performance of a laparosocopic task. Surg Endosc 17:1481-1484

34. Wetzel CM, Black SA, Hanna GB et al (2010) The effects of stress and coping on surgical performance during simulations. Ann Surg 251(1):171-176

35. Yule S, Flin R, Paterson-Brown S, Maran N (2006) Non-technical skills for surgeons in the operating room: a review of the literature. Surgery 139:140-149

36. Undre S, Sevdalis N, Healey AN, Darzi SA, Vincent CA (2006) Teamwork in the operating theatre: Cohesion or confusion? J Eval Clin Pract 12:182-189

37. Arora S, Sevdalis N, Nestel D, Woloshynowych M, Darzi A, Kneebone R (2010) The impact of stress on surgical performance: a systematic review of the literature. Surgery 147(3):311-330 